Invited paper

\title{
Lymphatic filariasis in the Eastern Mediterranean Region: current status and prospects for elimination
}

\author{
M. El Setouhy ${ }^{1}$ and R.M.R. Ramzy ${ }^{1}$
}

\begin{abstract}
SUMMARY Lymphatic filariasis (LF) represents a major public health problem in tropical and subtropical regions of the world. The disease is endemic or suspected in several countries of the Eastern Mediterranean Region. Recent advances in diagnosis and therapy led the World Health Assembly to pass a resolution in 1997 calling for "the elimination of lymphatic filariasis as a public health problem." The elimination strategy is based on rounds of mass drug administration of an annual single-dose of combined drug regimens for 5-6 consecutive years. Subsequent steps included formation of a Regional Programme Review Group to orient national LF control programmes towards the concept of elimination, provide advice, review each national plan of action and review annual reports. To date, Egypt and the Republic of Yemen have active national LF elimination programmes, however, elimination activities in the Republic of Yemen are still restricted to certain identified endemic regions. Other countries in the Region are on their way to verifying the situation and if LF is proved to be endemic, will start mapping endemic localities. This review sheds light on the status of LF elimination activities in the Region and highlights some of the major accomplishments.
\end{abstract}

\section{Introduction}

Lymphatic filariasis (LF), also known as elephantiasis, is a major disease of tropical and subtropical regions worldwide. LF is endemic in 80 countries, it is estimated that 120 million people are infected, with one third of them suffering from chronic manifestation of the disease. One billion more individuals are at risk of acquiring the infection [1].

Lymphatic filariasis is a mosquito-borne parasitic disease caused by three nematode worms of the family Filariidae: Wuchereria bancrofti, Brugia malayi and B. timori. Wuchereria bancrofti is responsible for $90 \%$ of worldwide infections, with $9 \%$ caused by $B$. malayi in southeast and east- ern Asia, whereas 1\% result from infection with B. timori in the Pacific region [2].

Bancroftian filariasis is caused primarily by adult worms (known as macrofilariae) that live in the lymphatic vessels. Female worms release embryonic microfilariae (MF) that in many endemic areas are characterized by nocturnal periodicity and thus circulate in the peripheral blood at night (21.00-02.00). The disease is transmitted by Anopheles, Culex and to a lesser extent by Aedes and Mansonia mosquito species. When mosquito vectors feed on infected subjects, they ingest MF along with their blood meal. In the vector, MF develop into infective larvae within 10-15 days. Mosquitoes transmit the infection from person to person. Infective larvae enter the human

${ }^{1}$ Research and Training Centre on Vectors of Diseases, Ain Shams University, Cairo, Egypt. 
skin through the wound made by the biting mosquito, moult and develop into adult worms in the afferent lymphatic vessels, causing severe distortion of the lymphatic system. Adult Wuchereria are often lodged in the lymphatics of the spermatic cord, causing scrotal damage and swelling. Elephantiasis (painful, disfiguring swelling of the limbs) is a classic sign of late-stage disease.

Traditionally, efforts to control LF generally included the 12-day diethylcarbamazine (DEC) treatment regimen $(6 \mathrm{mg} / \mathrm{kg}$ per day) recommended by the World Health Organization (WHO) or induced modifications to this regimen (e.g. single-dose DEC and DEC-fortified salt). Such efforts were supplemented in some areas by vector control [3].

\section{The World Health Organization strategy for lymphatic filariasis elimination}

Two primary biological characteristics of bancroftian parasites render transmission of infection insufficient: the lack of any animal reservoir host and no amplification of the parasite within the mosquito vector. Moreover, a new concept of disease elimination has resulted from recent fundamental developments in diagnostic methods and therapies, including improved diagnosis by the immunochromatographic diagnostic test (ICT), an antigen capture card format that detects filarial antigens in whole blood taken during daytime. The ICT card test has been proved to be sensitive, specific and visually readable within a few minutes directly in the field, rendering it a powerful tool for mapping LF endemic areas $[4,5]$. Furthermore, it is now recognized that the annual single-dose regimen of new microfilaricidal (and to a lesser extent macrofilari- cidal) drug treatment combinations (albendazole + DEC or albendazole + ivermectin) is safe for community-wide control programmes aiming to interrupt transmission of infection, and these combinations impressively reduce MF by $99 \%$ for a full year [6]. Such developments prompted the World Health Assembly to pass a resolution in 1997 (WHA 50.29) calling for "the elimination of lymphatic filariasis as a public health problem".

Consequently, WHO developed a new strategy and the Global Programme for Elimination of Lymphatic Filariasis was initiated with the aim of eliminating LF as a public health problem by the year 2020 . The programme has two principal goals: to interrupt transmission of infection and to alleviate and prevent both the suffering and disability caused by the disease [7]. Almost as important, however, is the necessity to achieve these goals in a cost-effective, socially-responsible manner, ensuring appropriate health and economic benefits [8]. The WHO efforts were greatly supported and enhanced by the announcement by GlaxoSmithKline in 1998 of its donation of specially manufactured (chewable and fruit flavoured) albendazole tablets needed for the global LF elimination programme free of charge.

To interrupt transmission of the infection, the essential strategy is to treat the entire at-risk population for a period long enough to ensure that MF levels in the blood remain below those necessary to sustain transmission by mosquitoes. For the yearly single-dose, 2-drug regimens are advocated (albendazole $400 \mathrm{mg}+$ DEC 6 $\mathrm{mg} / \mathrm{kg}$ or albendazole $400 \mathrm{mg}+$ ivermectin $200 \mathrm{mcg} / \mathrm{kg}$ ) in areas where onchocerciasis is co-endemic with bancroftian filariasis [9], this period has been estimated to be 4-6 years, corresponding to the reproductive lifespan of the parasite.

المجلة الصحية لشرق المتوسط، منظمة الصحة العالمية، المجلد التاسع، العدد ع، ب... 
To alleviate the suffering caused by the disease, the new strategy is aimed at management of the consequences of LF infection, particularly the lymphoedema (swelling of upper and/or lower limbs), elephantiasis and lymphoceles (scrotal swellings) and operated cases of lymphoceles or hydroceles. It is now evident that excessive local hygiene supplemented with antibiotics to inhibit bacterial super-infection can prevent or even reverse the lymphoedema and elephantiasis consequential to filarial infection.

\section{Lymphatic filariasis endemic countries in the Eastern Mediterranean Region}

It is estimated that approximately 0.4 million LF infected individuals, representing $<1 \%$ of the LF global burden, live in countries of the Eastern Mediterranean Region [2]. The disease is known to be focally endemic in 3 countries: Egypt, Sudan and the Republic of Yemen, whereas the LF situation in Djibouti, Oman, Pakistan, Saudi Arabia and Somalia is currently uncertain. However, clinical cases have been reported in Oman, Pakistan, Saudi Arabia and Somalia. In the countries of the Region, LF is entirely caused by W. bancrofti transmitted primarily by Culex mosquitoes in mostly rural and semi-urban areas.

A Regional Programme Review Group for elimination of lymphatic filariasis was assembled in 2001 to meet on a yearly basis for reviewing and evaluating implementation and progress of lymphatic filariasis elimination activities in endemic countries. It also identifies operational problems and research issues, discusses the detailed strategic planning of regional and country programmes, and identifies challenges and solutions in scaling-up of LF elimination activities.

During the Regional Programme Review Group meeting in 2002, a decision was made to scale up mapping activities in the Republic of Yemen and to initiate such activities in Oman, Saudi Arabia and Sudan in 2003. Mapping activities will be conducted in Djibouti, Pakistan and Somalia in 2004.

Funds to support LF elimination activities in the countries of the Eastern Mediterranean Region have been made available through several funding agencies. In particular, the Arab Fund for Economic and Social Development, located in Kuwait, donated a generous contribution aimed at helping countries to start LF elimination programmes.

\section{Current status of lymphatic filariasis elimination programmes in endemic countries of the Eastern Mediterranean Region}

Egypt

The population of Egypt (administratively consisting of 26 governorates) currently amounts to about 68 million, of which more than $60 \%$ reside in the densely populated governorates of the Nile Delta. In Egypt, nocturnally periodic LF caused by W. bancrofti infection has been endemic in rural areas for a long time [10]. Culex pipiens is the main vector mosquito and is extremely abundant throughout country. The disease has a focal distribution, causing a major public health problem in 6 governorates in the Nile Delta as well as in Giza and Assuit governorates in Upper Egypt. However, due to sustained control measures by the Ministry of Health and Population (MOHP), most endemic villages have low infection prevalence rates and intensities [11]. 
Egypt, in developing a national programme to eliminate LF as a public health problem, with the particular aim of reducing microfilaria prevalence rates to less than $1 / 1000$, was one of the first countries to join the WHO global effort. The programme is based on mass drug administration (MDA) of annual single-doses of DEC $(6 \mathrm{mg} / \mathrm{kg})$ in combination with albendazole (400 mg). The village level was chosen as the implementation unit for MDA. All villages with antigen prevalence rates of $1 \%$ or more were included in the programme with the goal of achieving an MDA coverage rate of about $80 \%$ of the total population in the target villages. Children under 2 years of age and pregnant women were excluded.

The programme depended on the welldeveloped network of rural health centres, which are part of the MOHP infrastructure. It also included a training component for physicians and nurses working at the rural health centres of the target villages and participating in the implementation of MDA. Social mobilization included meetings with local village leaders, distribution of pamphlets and posters, and short radio and television broadcasts for the dissemination of information about the LF elimination programme in order to create public awareness and facilitate community participation.

To date, the national programme has successfully completed 3 rounds of MDA in 161 (2000) and 179 (2001 and 2002) endemic villages. The total number of treated persons increased from 1759553 in the year 2000, to 2305724 in 2001 and 2426968 in 2002. This was due to a number of factors: more implementation units added in 2001, increased community participation and population growth. The MOHP estimated that the overall MDA coverage rate in 2000 and 2001 reached 96.6\% of the target population and $96.8 \%$ in 2002 . In concordance with the MOHP data, an independent evaluation sponsored by EMRO and carried out following the third round in 7 governorates revealed an overall MDA coverage rate of $87.6 \%$.

Adverse events after the first MDA were rare and mostly of mild to moderate severity, the most frequently observed being fever, headache, and myalgia. These symptoms, believed to be mainly due to dying worms, usually resolved within 2 to 3 days. Adverse reactions following the second and third rounds of MDA were greatly reduced compared to those observed following the first round.

Spot surveys in several localities following each round of MDA showed a remarkable impact of the drug combination on MF prevalence rates and intensities. In a recent study in four localities, it was observed that overall MF prevalence decreased by $75 \%$ (from $8.0 \%$ to $2.0 \%$ ) and the median MF levels in MF-positive subjects fell by $79 \%$ (from $42 / \mathrm{mL}$ to $9 / \mathrm{mL}$ ). The MF/mL/population dropped by $94 \%$ (from 13.0 to 0.8 ) after two rounds of MDA [12].

As part of the MOHP active surveillance, 54 villages, 2 villages per district, representing the 8 endemic governorates were surveyed in August 2003. These sentinel villages were chosen because they had relatively high baseline MF prevalence rates (before the national elimination programme) and/or low coverage rates in the last MDA. A total of 500 inhabitants/village were surveyed at night for MF (thick smears) by MOHP personnel. Of the 54 villages studied , 7 (13\%) were reported to have at least one MF positive subject. The MF prevalence in positive villages ranged between $0.2 \%$ and $1.0 \%$.

On the entomologic side, a recent study showed that rates and intensities of MF ingestion and infective larvae production by mosquitoes fed on smear-negative treated

المجلة الصحية لشرق المتوسط، منظمة الصحة العالمية، المجلد التاسع، العدد ؟، ب... 
subjects were significantly lower than female mosquitoes fed on smear-positive treated subjects. Therefore, the authors concluded that smear rates of zero could be a practical goal of elimination programmes [13].

We emphasize that so far the national LF elimination programme in Egypt has succeeded in maintaining high MDA coverage rates. If such rates can be sustained for the whole of the planned period, it is likely that the MDA programme will lead to the elimination of filariasis as a public health problem in Egypt.

\section{Sudan}

Lymphatic filariasis is evidently endemic in Sudan based on previous published [14] and unpublished data of scattered spot surveys and hospital records (lymphoedema and/or hydrocele). Of the 26 Sudanese states, 12 states are considered LF-endemic areas. The disease is focally endemic in the following southern states: Upper Nile, Unity, Jongli, Eastern Equatoria, Bahr Al Jabal, Western Equatoria, El Buheirat, Warab, Western Bahr El Ghazal and Northern Bahr El Ghazal; and Darfur and Blue Nile states in central Sudan. In addition, 5 more states (Sinnar, Gedaref, Northern, El Gezira and Khartoum) are suspected to be endemic for LF. However, since no recent systematic epidemiological surveys have been done, other states cannot be considered free of LF.

Mapping of LF in Sudan is currently hampered because of the conflict in parts of the country. Therefore there are certain areas that cannot be accessed for epidemiological surveys. Consequently, the true epidemiological picture remains unclear, and mapping of LF remains an essential first step to a systematic approach to eliminating the disease from Sudan.

Nevertheless, in 2003, a pilot survey in 4 states (Blue Nile, Sinnar, Western Bahr El
Ghazal and Bahr El Jebel) was carried out for rapid assessment of the LF situation using the questionnaire approach and a limited number of ICT cards to confirm the presence of active filariasis infection. Prior to the survey, a training workshop for medical personnel was conducted to develop the questionnaire (supplemented with pictures of chronic cases) in local languages and to give training on correct performance of the ICT card test. Trained teams interviewed key informants, including community heads, sultan, schoolteachers and medical staff whenever possible.

The survey in Sinnar state revealed many cases in people who had migrated from Blue Nile state. Surprisingly, of 21 villages surveyed in Blue Nile state, 12 (57.1\%) villages were proved LF endemic by the ICT test (1-2 cards/village). Out of 28 villages in the state of Bahr El Ghazal, key informants in 18 (64.3\%) villages reported 21 lymphoedema and 86 hydrocoel cases. The questionnaire survey in 39 villages in Bahr El Jebel state discovered 84 people with lymphoedema and 40 with hydrocoel, however, only 2 villages were confirmed endemic by testing a limited number of subjects with the ICT test.

In conclusion, this primary survey, with such limited resources, documented the existence of several LF endemic areas in Sudan and should be continued in order to map other endemic localities by lot quality assurance survey (LQAS) using ICT cards in the same states. Other states (Upper Nile, Equatoria, Southern Darfur, and Southern Kordufan) should also be considered whenever possible.

\section{Republic of Yemen}

The Republic of Yemen, with a population of about 20 million people, is an Asian-Arab country occupying the southwest tip of the Arabian Peninsula on the Red Sea, and extends along the southern part of the Arabian 
Peninsula on the Gulf of Aden and the Indian Ocean.

Administratively, the country is divided into 20 governorates, each divided into districts. These are further divided into subdistricts and then into villages.

The Republic of Yemen is a known endemic country for onchocerciasis. However, the LF situation was uncertain since few lymphoedema cases, without confirmed laboratory diagnosis, were recognized. Thus, the transmission of the disease was possible but nor verified. The leprosy mission, a unit related to the Yemeni Ministry of Public Health, is also responsible for onchocerciasis control and prevention. As the mission has a well-developed network of clinics distributed throughout the country, it was chosen as the body responsible for the National Programme for Elimination of LF.

In 2000, as a first step to locating possible endemic localities, questionnaires supplemented with pamphlets showing photos of cases with chronic manifestation (lymphoedema and/or lymphoceles) were distributed to key informants, including government hospitals and local health authorities, as well as to community leaders in all governorates. Analysis of data gathered from the key informants along with the environmental data of these areas revealed that 13 subdistricts in 6 governorates are probable LF endemic areas whereas another 24 subdistricts in different governorates are possible endemic localities. Consequently, an LQAS using the ICT card test [10] was carried out in these suspected areas during 2001-2002. While 11 of the 13 suspected subdistricts had at least 1 positive card test, and therefore were eligible for MDA, none of the 24 subdistricts proved LF endemic by the LQAS.

Prior to MDA, training sessions for 634 workers at primary health centres were carried out. Social mobilization activities included meeting with community leaders and schoolteachers, distribution of health education materials, and TV spots and short radio interviews. For the national programme for elimination of LF, the subdistrict was chosen as the implementation unit for MDA. In 2002 an initial pilot MDA was implemented in Wisab subdistrict, Dhamar governorate (about 12800 inhabitants) and 2 subdistricts on Socotra island, Hadramout governorate (about 29000 inhabitants). The first round of MDA with a combined drug regimen of ivermictin (200 $\mathrm{mcg} / \mathrm{kg}$ ) and albendazole (400 mg) was carried out from house to house based on directly observed therapy by primary health centre distributors. Children under 5 years of age and pregnant women were excluded from MDA. The programme estimated an overall $85 \%$ MDA coverage rate (86\% in Socotra and $84 \%$ in Wisab).

An active surveillance was carried out on 1400 randomly selected persons in the areas covered to monitor the side effects of the mass therapy. No serious adverse experiences were notified and all side effects were mild, the main ones being development of swellings (3\%), fever $(0.9 \%)$ and headache $(0.9 \%)$. In addition, $76(5.4 \%)$ of the people surveyed reported passing different types of intestinal worms.

In 2003, the second round of MDA was carried out in the same areas and in 9 other areas proven endemic by LQAS using ICT cards. In the meantime, training of laboratory technicians was carried out to evaluate the impact of MDA in the areas covered using thick blood smears. The evaluation process in the 11 areas covered with MDA is scheduled for September-October 2003. Finally, to complete LF mapping in the Republic of Yemen by LQAS using ICT cards, 20 further suspected implementation units will be surveyed during January-March 2004.

المجلة الصحية لشرق المتوسط، منظمة الصحة العالمية، المجلد التاسع، العدد ع، با.. 


\section{Countries with uncertain lymphatic filariasis situation}

\section{Oman}

According to the latest general population census (2001), Oman has a population of approximately 2.5 million inhabitants, of which $26.3 \%$ are expatriates. Many of these expatriates come to work in Oman from LF endemic countries (e.g. India and Egypt) and had possibly lived in LF endemic areas.

Some of these expatriates may have acquired infection with $W$. bancrofti or $B$. malayi before their arrival in Oman, and therefore represent a significant source for LF transmission. In support of this view, a recent study has found an antigenaemia prevalence rate of $4.2 \%$, based on the ICT card test, in Indian expatriates living in Oman [15]. Lymphatic filariasis is a notifiable disease in the country and over the last decade the health authorities have identified 15 LF cases. Of these, 7 were Omanis who had lived for some time in LF endemic countries. While some of the 15 LF cases presented with elephantiasis, others had microfilareamia or a positive antibody test.

In an effort to verify LF status in 59 suspected wilayat (districts) during 2002, health authorities in Oman carried out a rapid assessment of the community burden of the disease using the questionnaire approach. Of 640 community leaders as key informants included in the survey, 23 (3.6\%) informants, representing 12 districts, reported that they had seen 1 or more cases with chronic manifestation (elephantiasis). Out of 930 physicians 21 (3.1\%) had observed cases of elephantiasis and/or hydrocele.
The next step is to conduct school surveys using the ICT card test in suspected LF endemic localities and to test representative blood samples from blood donors in areas considered free of LF.

In conclusion, sporadic cases of chronic LF exist in certain districts of Oman, however, as yet LF transmission may not represent a health threat. Health authorities in Oman are committed to joining the WHO global effort to eliminate the disease and taking the steps necessary to prepare Oman for certification as an LF-free country.

\section{Saudi Arabia}

Saudi Arabia, with an estimated population of approximately 24 million inhabitants (2003), is divided into 13 provinces for administrative purposes. Lymphatic filariasis was first reported in a few chronic cases from two areas, Asir and Jizan, in the 1970s. During the 1990s, several expatriates, mostly Indians, were found to be LF positive [16]. Recently, based on a questionnaire survey, a total of 51 clinical cases (15-20 years of age) with elephantiasis or hydrocele, although amicrofilaraemic, were identified from 3 areas Asir (44 cases), Jizan (4 cases) and Mecca (3 cases). Subsequently, a total of 34 laboratory technicians were trained to perform the ICT card test. However, due to a technical problem encountered at that time with the Binax ICT cards showing false positive results after 10 minutes, they could not be used to conduct school surveys in suspected endemic areas. Thus, serological evaluation of the current LF situation in Saudi Arabia was postponed, waiting for a modified version of the ICT cards. 


\section{References}

1. Ottesen EA. Major progress toward eliminating lymphatic filariasis. New England journal of medicine, 2002, 347:1885-6.

2. Michael E, Bundy DAP. Global mapping of lymphatic filariasis. Parasitology today, 1997, 13:472-6.

3. Ottesen E. The global programme to eliminate lymphatic filariasis. Tropical medicine and international health, 2000 , 5:591-4.

4. Weil GJ, Lammie P, Weiss N. The ICT filariasis test: A rapid-format antigen test for diagnosis of bancroftian filariasis. Parasitology today, 1997, 13:401-4.

5. Ramzy RM et al. Field evaluation of a rapid-format kit for diagnosis of bancroftian filariasis in Egypt. Eastern Mediterranean health journal, 1999, $5: 880-7$

6. Ismail $\mathrm{M}$ et al. Prolonged clearance of microfilaraemia after multiple high doses of ivermectin or diethylcarbamazine. Transactions of the Royal Society of Tropical Medicine and Hygiene, 1996, 90:684-8.

7. Preparing and implementing a national plan to eliminate lymphatic filariasis in countries where onchocerciasis is not co-endemic. Geneva, World Health Organization, 2002 (WHO/CDC/CPE/2000. 15).

8. Molyneux DH, Zagaria N. Lymphatic filarisis elimination: progress in global programme development. Annals of tropical medicine and parasitology, 2002, 96:S15-40.

9. Preparing and implementing a national plan to eliminate lymphatic filariasis in countries where onchocerciasis is coendemic. Geneva, World Health Organization, 2002 (WHO/CDC/CPE/2000.16).

10. Southgate BA. Bancroftian filariasis in Egypt. Tropical diseases bulletin, 1979, 76:1045-68.

11. Weil GJ et al. Iongitudinal study of bancroftian filariasis in the Nile Delta of Egypt: baseline data and one-year follow-up. American journal of tropical medicine and hygiene, 1999, 6: 53-8.

12. Ramzy RMR et al. Filariasis elimination in Egypt: impact of low microfilaraemics as sources of infection for mosquitoes. Eastern Mediterranean health journal, 2003, 9(4):863-72.

13. Farid HA et al. Relationships between Wuchereria bancrofti microfilaria counts in human blood and parasite uptake and maturation in Culex pipiens, with observations on the effects of diethylcarbamazine treatment on these parameters. American journal of tropical medicine and hygiene, 2003, 68(3):286-93.

14. Satti MH, Abdel Nur O. Bancroftian filariasis in the Sudan. Bulletin of the World Health Organization, 1974, 51:314-5.

15. Scrimgeour EM et al. Bancroftian filariasis in residents of Oman. Acta tropica, 2001, 79(3):241-4.

16. Omar MS et al. Field evaluation of two diagnostic antigen tests for Wuchereria bancrofti infection among Indian expatriates in Saudi Arabia. Southeast Asian journal of tropical medicine and public health, 2000, 31(2):415-8. 